\title{
The Anti-Inflammatory and Cytoprotective Efficiency of Curvularin, a Fungal Macrolactone against Lipopolysaccharide-Induced Inflammatory Response in Nucleus Pulposus Cells: An In Vitro Study
}

\author{
Rajkiran Reddy Banala ${ }^{1,{ }^{*}}$, Satish Kumar Vemuri ${ }^{1,{ }^{*}}$, Sherline $\mathrm{EV}^{3}$, Gurava Reddy AV$^{1}$, Subbaiah GPV ${ }^{1,2}$ \\ ${ }^{I}$ Sunshine Medical Academy of Research and Training, Sunshine Hospitals, Secunderabad, India \\ ${ }^{2}$ Department of Spine Surgery, Star Hospitals, Hyderabad, India \\ ${ }^{3}$ Department of Microbiology, St. Francis College for Women, Hyderabad, India
}

\begin{abstract}
Study Design: Developing an in vitro model for assessing the anti-inflammatory properties of curvularin.
Purpose: To evaluate the efficacy of natural fungal macrolactone as a therapeutic drug against lipopolysaccharide (LPS)-induced inflammation in primary human nucleus pulposus cells (NPCs) in vitro.

Overview of Literature: Lumbar disk disease is a common cause of lower back pain (LBP) and sciatica. It is an established fact that inflammation, rather than mechanical compression on the nerve root, plays a role in the cause of LBP and sciatica. Current treatment options for reducing inflammation are either nonsteroidal anti-inflammatory drugs or steroids, prolonged use of which can potentially lead to adverse effects such as gastrointestinal disturbances and renal and cardiac issues. Hence, there is a need for better antiinflammatory drugs with no or minimal complications for treating inflammation-induced LBP and sciatica. Curvularin (Cur), a fungal macrolactone, is known for its anti-inflammatory activity, but nothing is known about its impact on inflammation due to disk pathologies.

Methods: Primary NPCs were cultured and characterized by flow cytometry and immunocytochemistry using the CD24 antibody and treated with $10 \mu \mathrm{g} / \mathrm{mL}$ LPS for 36 hours and then treated with Cur, betamethasone, and dexamethasone ( $10 \mu \mathrm{g} / \mathrm{mL})$ for 48 hours, after which cell cycle analysis, cell viability assay, and gene expression studies (quantitative polymerase chain reaction [PCR] and quantitative real-time-PCR) were conducted. The NPCs treated with Cur downregulated the expression of pro-inflammatory cytokines (tumor necrosis factor- $\alpha$, interleukin [IL]-1 $\beta$, and IL-6); matrix metalloproteinases (MMPs; MMP-2 and MMP-3), ADAMTS; and apoptotic marker (cytochrome c).

Results: In our study, Cur-treated cells showed enhanced expression of collagen 9A1 and insulin-like growth factor receptor 1, indicating the recovery of NPCs from inflammatory assault.

Conclusions: Based on observations, the anti-inflammatory properties of Cur render it an excellent drug molecule for treating disk degeneration nonsurgically, by direct injection into spinal disks when treating LBP and sciatica.
\end{abstract}

Keywords: Intervertebral disc degeneration; Macrolactone; Curvularin; Lipopolysaccharide; Inflammation; Cytokines; Nonsteroidal anti-inflammatory drugs

Received Sep 3, 2019; Revised Oct 23, 2019; Accepted Nov 4, 2019

Corresponding author: Subbaiah GPV

Department of Spine Surgery, Star Hospitals, Road no. 10, Banjara Hills, Hyderabad-500034, Telangana, India

Tel: +91-040-44550000, Fax: +91-040-27890091, E-mail: drgpvsubbaiahgoli@gmail.com

*These authors contributed equally to this work. 


\section{Introduction}

One of the common causes of lower back pain (LBP) and sciatica is intervertebral disc degenerative (IVDD) pathologies $[1,2]$, a multifactorial process with complex biochemical cascade, due to the involvement of genetic and environmental factors in its etiology [2]. Perineural inflammation, rather than mechanical compression, has proven to be the primary cause of LBP and sciatica [3-5]. Numerous anti-inflammatory drugs are used in the treatment of LBP and sciatica but with limited success, leading to a significant economic burden on the global population [6]. Continuing efforts are being made by various scientific groups to find alternative anti-inflammatory drugs with better efficacy and safety.

A focus on phytochemicals denotes good anti-inflammatory properties, which can potentially reduce LBP and sciatica. Known phytocompounds, such as curcumin [5], resveratrol (3,5,4'-trihydroxy-trans-stilbene) [6], naringin [7], naringenin [7], quercetin [7,8], simvastatin [9], atorvastatin, lovastatin [9], and others, are known for their anti-inflammatory properties that downregulate interleukins (IL-1 $\alpha$, IL- $\beta$, IL-6, IL-18), tumor necrosis factor- $\alpha$ (TNF- $\alpha$ ), and aggrecanases and collagenases (matrix metalloproteases [MMPs] and ADAMTS) in nucleus pulposus cells (NPCs) [10-16]. These flavonoid-based drugs can be used individually or in combinations to control inflammation and to potentially regenerate intervertebral discs.

The present study focuses on inflammation-induced LBP and sciatica, which are treated symptomatically rather than by regenerative or curative therapies. Globally, researchers have used different inflammatory cytokines (i.e., IL-1 $\beta$, IL- $1 \alpha$, TNF- $\alpha$, and IL-6) to induce inflammation in human NPCs, reporting increased expression of MMP-1-MMP-3, MMP-9, MMP-13, ADAMTS-4, and ADAMTS-5 and decreased expression of aggrecan, collagen types I and II, and SOX6 and SOX9 $[6,9,12$ 15,17-20] (Table 1). In the current study, we used LPS to induce inflammation in NPCs. Our study aims to evaluate the anti-inflammatory and regenerative efficiency of curvularin (Cur), a known natural fungal macrolactone $[16,21,22]$, against LPS-induced inflammation in primary human NPCs, by altering the expression profiles of proinflammatory and metalloprotease genes in vitro. To our knowledge, no studies have been published on Cur that show it as having anti-inflammatory and cytoprotective properties for NPCs; hence, our study will be the first to evaluate Cur in this context.

\section{Materials and Methods}

To achieve the aims of this study, Cur (isolated from Penicillium citrinum, C16H20O5, cat no. SML0772) and lipopolysaccharide (LPS) (isolated from Escherichia coli, O111:B4, cat no. L3024) were purchased from SigmaAldrich (Bangalore, India). The purity of the compounds was $\geq 95 \%$ (derived via high-performance liquid chromatography). Cur and LPS were reconstituted in dimethyl sulfoxide (Sigma-Aldrich) to yield a stock solution of 2 $\mathrm{mg} / \mathrm{mL}$. Dulbecco's Modified Eagle Medium/nutrient mixture F12 Ham (DMEM-F12, 1:1 mixture), 10\% fetal bovine serum (FBS), $0.1 \%$ collagenase type I, an antibiotic antimycotic solution $100 \times$ with 10,000 units of penicillin, $100 \mathrm{mg}$ streptomycin and $25 \mu \mathrm{g}$ amphotericin B per mL

Table 1. Overview of inflammatory mediators used for inducing inflammation in varied NPCs and expression profile of cytokines, metalloproteases, and so forth

\begin{tabular}{|c|c|c|c|}
\hline Reference & Stimulator & Models & Expression \\
\hline $\begin{array}{l}\text { Wuertz et al. [6] (2011); Le Maitre et al. [12] (2005); Yu } \\
\text { et al. [13] (2009); Wang et al. [14] (2011); Zhao et al. } \\
\text { [15] (2011); Shen et al. [17] (2003) }\end{array}$ & $\mathrm{IL}-1 \beta$ & Human NPC & $\begin{array}{l}\text { Upregulated MMP's }(1,3,9 \text {, and } 13) \text {, ADAMTS (4 and 5) and down- } \\
\text { regulated aggrecan, collagen type I \& type II, SOX (6 and 9) }\end{array}$ \\
\hline Shen et al. [18] (2018) & LPS & Rats NPC & $\begin{array}{l}\text { Upregulated TNF- } \alpha, \text { IL-1B, PGE2, NO, iNOS, COX-2, p-IkB, p-P65; down- } \\
\text { regulated collagen II and aggrecan }\end{array}$ \\
\hline Mancino et al. [19] (2011) & LPS & Bovine IVD cells & IL-1ß, IL-6, and IL-10 upregulated; collagen 1 and 2 down-regulated \\
\hline Li et al. [9] (2015) & LPS & Rat NPC & $\begin{array}{l}\text { Upregulated ADAMTS4, ADAMTS5, MMP-3, MMP-13, PGE2, COX-2, and } \\
\text { INOS; down-regulated aggrecan and collagen II }\end{array}$ \\
\hline Kong et al. [20] (2018) & LPS & Rat NPC & $\begin{array}{l}\text { Upregulated MMP-3, MMP-13, ADAMTS4, ADAMTS5, TNF- } \alpha \text {, IL-1, IL-6, } \\
\text { and PGE2; down-regulated miR-194, aggrecan, and collagen II }\end{array}$ \\
\hline
\end{tabular}

NPCs, nucleus pulposus cells; IL, interleukin; MMPs, matrix metalloproteinases; LPS, lipopolysaccharide; TNF, tumor necrosis factor; PGE2, prostaglandin E2; NO, nitric oxide; iNOS, inducible nitric oxide synthase; COX-2, cyclooxygenase-2; IVD, intervertebral disc. 
in $0.9 \%$ saline, $0.25 \%$ trypsin-ethylenediaminetetraacetic acid, centrifuge tubes (Tarsons, Kolkata, india; cat no. 546041), $100 \mu \mathrm{m}$ nylon mesh (Himedia Laboratories, Mumbai, India; reference TCP183), and all other chemicals and reagents were analytical or culture grades, purchased from Himedia Laboratories and Sigma-Aldrich.

\section{In vitro studies: culture of human nucleus pulposus cells}

Intervertebral disc tissue from the lumbar spine was collected from patients undergoing interbody fusion surgery for degenerative disk disease (institutional ethical approval was secured prior to collection-SS/2015/IEC127). Disk tissue was immediately collected in $20 \mathrm{~mL}$ saline supplemented with an antibiotics solution and transported to a laboratory at $4^{\circ} \mathrm{C}$. All procedures were conducted in a sterile environment. Tissue samples were repeatedly washed in a phosphate buffer saline (PBS) solution, and then the NPCs were separated by slicing the disk tissue into $1 \mathrm{~mm}^{3}$ segments in a moist environment and digested with $0.1 \%$ collagenase type I at $37^{\circ} \mathrm{C}$ for 4 hours, with intermittent shaking at 30 -minute intervals. Following this, the reaction was stopped with FBS. The digested mixture was then filtered through a sterile $100 \mu \mathrm{m}$ nylon mesh and centrifuged at 1,500 rpm for 5 minutes to collect cell pellets. The cells were repeatedly washed in a PBS solution and resuspended in a DMEM-F12 medium containing $10 \%$ FBS and antibiotics (prostate specific antigen) and seeded with a cell concentration density of $1 \times 10^{5} / \mathrm{mL}$ into a $75 \mathrm{~cm}^{2}$ flask. Cells were maintained in culture flasks at $37^{\circ} \mathrm{C}$ in a $5 \% \mathrm{CO}_{2}$ atmosphere, and culture media was changed 2 to 3 times a week. NPCs were used for further experimentation at passage three $[23,24]$.

\section{Characterization of human nucleus pulposus cells by flow cytometry}

Degenerated human NPCs were cultured in a T75 culture flask in the presence of DMEM-high glucose and 10\% FBS; $1 \times$ penicillin-streptomycin antibiotics were allowed to grow to $70 \%$ to $80 \%$ confluence, trypsinized, and resuspended in $300 \mu \mathrm{L}$ PBS. The cells were characterized by examining the presence of CD24 markers (positive markers) using standard flow cytometry methods [23].

\section{Treatment with lipopolysaccharide and curvularin}

Cultured NPCs $\left(5 \times 10^{4} / \mathrm{mL}\right)$ were seeded in three 12 -well cell culture plates for up to 72 hours prior to experimentation. The control group received no treatment (Table 2). The NPCs were treated with $10 \mu \mathrm{g} / \mathrm{mL}$ LPS for 36 hours before cells were treated with $10 \mu \mathrm{g} / \mathrm{mL}$ Cur, and betamethasone (Beta) and dexamethasone (Dexa) was individually added to respective group wells and in combination (Cur, Beta, Dexa in a 1:1:1 ratio) for 48 hours before harvesting total RNA [24] (Table 2).

\section{Cell cycle studies by flow cytometry}

Degenerated human NPCs were cultured in $60 \mathrm{~mm}$ dishes and initially treated with LPS $(10 \mu \mathrm{g} / \mathrm{mL})$ for 24 hours. Later, anti-inflammatories Cur $(10 \mu \mathrm{g} / \mathrm{mL})$ and Dexa (10 $\mu \mathrm{g} / \mathrm{mL})$ and a combination of Cur and Dexa $(10 \mu \mathrm{g} / \mathrm{mL})$ were added to cells, harvested after 36 hours, prepared for cell proliferation probing with propidium iodide for 15 to 20 minutes in the dark at room temperature, and estimated using an Attune NxT flow cytometer (Applied Biosystems, Foster City, CA, USA) as per manufacturer's instructions $[25,26]$.

\section{Cell viability assay/MTT assay}

An MTT assay was conducted to establish the cytotoxicity of drugs and was measured based on the number of viable cells. Normal cells (i.e., human embryonic kidney [HEK] cell lines) were plated in 96 well plates. Cells were individually treated with vehicle control or Cur, Beta, or Dexa and in combinations (Cur, Beta, Dexa) for 36 hours. The reason for this selection was to avoid unwanted cytotoxicity in normal cells. After 36 hours, cell viability was analyzed using the MTT assay, based on published protocols, and absorbance was recorded at $475 \mathrm{~nm}$, with reference to

Table 2. Experimental groups

\begin{tabular}{|c|c|}
\hline Groups & Treatment \\
\hline Control (negative control) & No treatment \\
\hline Lipopolysaccharide & $10 \mu \mathrm{g} / \mathrm{mL}$ \\
\hline Cur & $10 \mu \mathrm{g} / \mathrm{mL}$ \\
\hline Beta & $10 \mu \mathrm{g} / \mathrm{mL}$ \\
\hline Dexa & $10 \mu \mathrm{g} / \mathrm{mL}$ \\
\hline Combination & Cur-Bet-Dexa in 1:1:1 ratio \\
\hline
\end{tabular}

Cur, curvularin; Beta, Betamethasone; Dexa, Dexamethasone. 
$660 \mathrm{~nm}[25,26]$.

\section{Gene expression studies: RNA isolation, cDNA syn- thesis, and real-time quantitative polymerase chain reaction}

After 48 hours, NPCs were trypsinized, and total RNA was isolated using the Nucleopore Mini Kit (Genetix Biotech, New Delhi, India). The concentration of RNA was determined using NanoDrop; $50 \mathrm{ng} / \mathrm{mL}$ of total RNA was used for cDNA synthesis using a commercial cDNA synthesis kit (Verso cDNA Synthesis Kit; Thermo Fischer Sci- entific, Waltham, MA, USA), and gene expression studies (quantitative polymerase chain reaction $[\mathrm{PCR}]$ ) were conducted using a SimpliAmp Thermal Cycler (Applied Biosystems) and real-time PCR (QuantStudio 5, Applied Biosystems, Thermo Fischer Scientific).

All PCR products were visualized on a 1.5\% agarose gel with $0.5 \mathrm{mg} / \mathrm{mL}$ ethidium bromide. Photographs were taken using the Gel Documentation System (E Gel Imager; Life Technologies, Carlsbad, CA, USA). The cDNAs were used for studying the gene expression of interleukins (IL-1 $\beta, 1 L-6$, IL-2, IL-10), TNF- $\alpha$, MMPs (MMP-2, MMP3, ADAMTS-5), mineralization and adhesion markers

Table 3. List of primer sequences used for gene expression studies

\begin{tabular}{|c|c|c|c|c|}
\hline Serial no. & Gene name & Sequence $\left(5^{\prime}-3^{\prime}\right)$ & Time (sec) & Functions \\
\hline 1 & TNF- $\alpha$ & $\begin{array}{l}\text { Forward: AAGCACACTGGTTTCCACACT } \\
\text { Reverse: TGGGTCCCTGCATATCCGTT } \\
\text { Product size: } 74 \text { bp }\end{array}$ & 58 & Inflammatory cytokine \\
\hline 2 & IL-1 $\beta$ & $\begin{array}{l}\text { Forward: AACAGATGAAGTGCTCCTTCCAGG } \\
\text { Reverse: TGGAGAACACCACTTGTT GCTCCA } \\
\text { Product size: } 390 \text { bp }\end{array}$ & 61 & Inflammatory cytokine \\
\hline 3 & $\mathrm{IL}-6$ & $\begin{array}{l}\text { Forward: ACTCACCTCTTCAGAACGAATTG } \\
\text { Reverse: CCATCTTTGGAAGGTTCAGGTTG } \\
\text { Product size: } 159 \text { bp }\end{array}$ & 61 & Pro-inflammatory cytokine \\
\hline 4 & IL-2 & $\begin{array}{l}\text { Forward: CATGTACAGCATGCAGCTCGCATCC } \\
\text { Reverse: CCACCACAGTTGCTGGCTCATCATC } \\
\text { Product size: } 390 \text { bp }\end{array}$ & 66 & Anti-inflammatory cytokine \\
\hline 5 & IL-10 & $\begin{array}{l}\text { Forward: GACTTTAAGGGTTACCTGGGTTG } \\
\text { Reverse: TCACATGCGCCTTGATGTCTG } \\
\text { Product size: } 390 \text { bp }\end{array}$ & 62 & Anti-inflammatory cytokine \\
\hline 6 & MMP-2 & $\begin{array}{l}\text { Forward: GATACCCCTTTGACGGTAAGGA } \\
\text { Reverse: CCTTCTCCCAAGGTCCATAGC } \\
\text { Product size: } 111 \mathrm{bp}\end{array}$ & 62 & Degradation of the disc matrix \\
\hline 7 & MMP-3 & $\begin{array}{l}\text { Forward: CGGTTCCGCCTGTCTCAAG } \\
\text { Reverse: CGCCAAAAGTGCCTGTCTT } \\
\text { Product size: } 285 \text { bp }\end{array}$ & 64 & Breakdown of extracellular matrix \\
\hline 8 & ADAMTS-5a) & $\begin{array}{l}\text { Forward: GAACATCGACCAACTCTACTCCG } \\
\text { Reverse: CAATGCCCACCGAACCATCT } \\
\text { Product size: } 106 \text { bp }\end{array}$ & 64 & Pathogenesis of human osteoarthritis \\
\hline 9 & IGF1R & $\begin{array}{l}\text { Forward: AGGATATTGGGCTTTACAACCTG } \\
\text { Reverse: GAGGTAACAGAGGTCAGCATTTT } \\
\text { Product size: } 79 \text { bp }\end{array}$ & 61 & Hypertrophy of muscle and tissue \\
\hline 10 & CYT-C & $\begin{array}{l}\text { Forward: TTCCGGGCAAAATGGCGAT } \\
\text { Reverse: CAAGGAAGAGGTAGTGTGGGC } \\
\text { Product size: } 179 \mathrm{bp}\end{array}$ & 58 & Apoptosis \\
\hline 11 & COL9A1 & $\begin{array}{l}\text { Forward: GGCAGTAGAGGAGAATTAGGACC } \\
\text { Reverse: GTTCACCGACTACACCCCTG } \\
\text { Product size: } 141 \text { bp }\end{array}$ & 64 & Delay onset of osteoarthritis \\
\hline 12 & GAPDH & $\begin{array}{l}\text { Forward: GGCTCTCCAGAACATCATCCCTGC } \\
\text { Reverse: GGGTGTCGCTGTTGAAGTCAGAGG } \\
\text { Product size: } 260 \text { bp }\end{array}$ & 66 & House keeping gene \\
\hline
\end{tabular}

TNF, tumor necrosis factor; IL, interleukin; MMPs, matrix metalloproteinases; IGF1R, insulin-like growth factor 1 receptor; CYT-C, cytochrome c; COL9A1, collagen 9 alpha 1; GAPDH, glyceraldehyde 3-phosphate dehydrogenas.

${ }^{\text {a) } A}$ disintegrin and metalloproteinase with thrombospondin motifs 5. 
(insulin-like growth factor 1 receptor [IGF1R]), death markers (cytochrome c [CYT-C]), and glyceraldehyde 3-phosphate dehydrogenase (GAPDH) as a housekeeping gene [24] (Table 3).

\section{Results}

\section{Culturing of degenerated human nucleus pulposus cells}

Degenerated human NPCs were successfully isolated from the disk, propagated, cultured for P0 to P3 generation, and characterized in the study after passage 3 . The cells were polygonal in shape, similar to chondrocytes (Fig. 1).

\section{Characterization of nucleus pulposus cells by flow cytometry and immunocytochemistry studies}

Fig. 2A illustrates the CD24-positive population (NPCs), where the M1 peak denotes unstained cells that were not positive for CD24, and the M2 peak denotes the cell population positively stained by CD24. Fig. 2B also demonstrates CD24-positive cells under fluorescence, which indicates that most of the population in isolated cells were NPCs.

\section{Treatment with curvularin, betamethasone, and dexamethasone}

Cultured NPCs $\left(5 \times 10^{4}\right.$ cells $\left./ \mathrm{mL}\right)$ were seeded in 12 well plates for up to 72 hours prior to the experiment. Once cell confluence reached $80 \%$, these cells were treated with LPS $(10 \mu \mathrm{g} / \mathrm{mL})$ for 36 hours; subsequently, NPCs were treated with Cur, Beta, and Dexa $(10 \mu \mathrm{g} / \mathrm{mL})$ for 48 hours individually and also in combination (Cur-Beta-Dexa in a 1:1:1 ratio). Treatments were effected in duplicate.

NPCs treated with LPS were lower in number and ex-
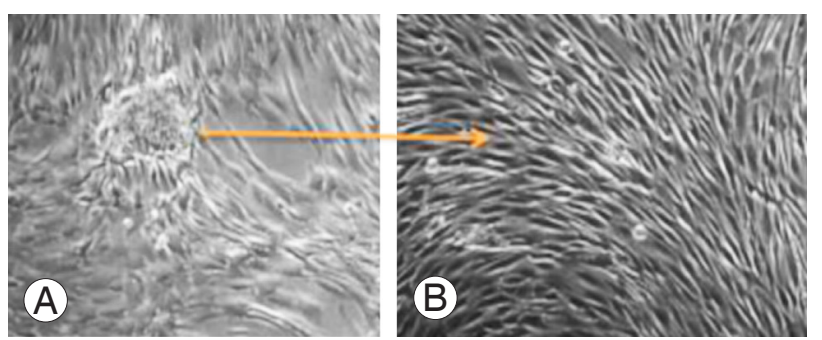

Fig. 1. (A, B) Culture of degenerated human nucleus pulposus cells. hibited degenerative morphology, whereas NPCs treated with anti-inflammatories or cytoprotectants showed better cell proliferation and cell morphology when individual and combined treatments were effected. Cur exhibited protective effects similar to commercial drugs.

\section{Cell cycle assay}

Degenerated human NPCs were initially treated with LPS $(10 \mu \mathrm{g} / \mathrm{mL})$ for 24 hours; later, the anti-inflammatories (Cur, $10 \mu \mathrm{g} / \mathrm{mL}$; Dexa, $10 \mu \mathrm{g} / \mathrm{mL}$; and a combination of Cur and Dexa, $10 \mu \mathrm{g} / \mathrm{mL}$ ) were added to cells and harvested after 36 hours. Untreated NPCs were considered a control. The cell proliferation analysis showed decreased cell proliferation in LPS-treated NPCs and enhanced cell proliferation in NPCs treated with anti-inflammatories. NPCs that received combination treatment showed more cell proliferation compared to individual anti-inflammatory treatment.

\section{Cell proliferation assay (MTT)}

We carried out MTT assay studies for the assessment of cell viability and cell proliferation efficiency of the HEK293 cell line; simultaneous treatment with LPS, Cur, Beta,
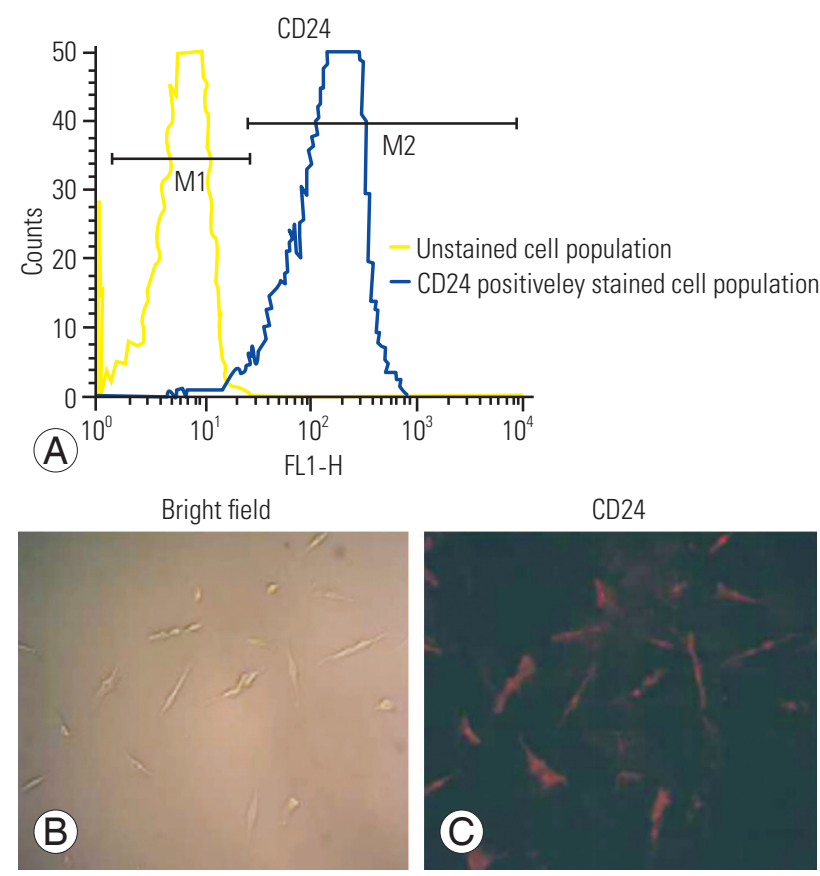

Fig. 2. (A-C) Flow cytometry and immunocytochemistry studies for the characterization of primary human nucleus pulposus cells using the CD24 antibody. 
and Dexa individually; and combinations of these after 36 hours of exposure (Fig. 3). Results of the MTT assay suggest that the Cur, Beta, and Dexa did not induce cytotoxicity in the HEK-293 (normal cell line), and cells proliferated normally when treated with similar dosages used during our study. No cytotoxicity was observed in any of the protective groups, but a lower number of cells was observed in the LPS-treated group. The $10 \mu \mathrm{g} / \mathrm{mL}$ Cur, Beta, and Dexa appeared to be effective and were found to be an optimum dose for promoting cell proliferation.

\section{Gene expression studies (real-time quantitative poly- merase chain reaction)}

The gene expression of TNF- $\alpha$, IL-1 $\beta$, IL-6, MMP-2, MMP-3, ADAMTS, CYT-C, IL-10, IL-2, IGF1R, collagen 9A1, and GAPDH was studied in a primary culture of NPCs, with induced inflammation using LPS. The NPCs were treated with LPS, LPS-Cur, LPS-Beta, LPS-Dexa, and with a combination (LPS-Cur-Bet-Dexa).

TNF- $\alpha$, IL- $1 \beta$, and IL- 6 are major pro-inflammatories, and their gene expression was significant in LPS-treated NPCs. NPCs treated with Beta and Dexa showed reduced expression of pro-inflammatories. The Cur and combination-treated NPCs showed no expression compared to LPS-treated NPCs (positive group). The untreated NPCs (control group) showed no expression of metalloproteases. Collagenases MMP-2 and MMP-3 degraded the extracellular matrix (ECM) and caused the loss of NPCs. MMP2 and MMP-3 gene expressions were higher in NPCs treated with LPS compared to treatment with protective agents. It was observed that cells treated with Beta and Dexa alone and in combination showed a lower expression of MMP-2 compared to LPS-treated cell. Cur was less

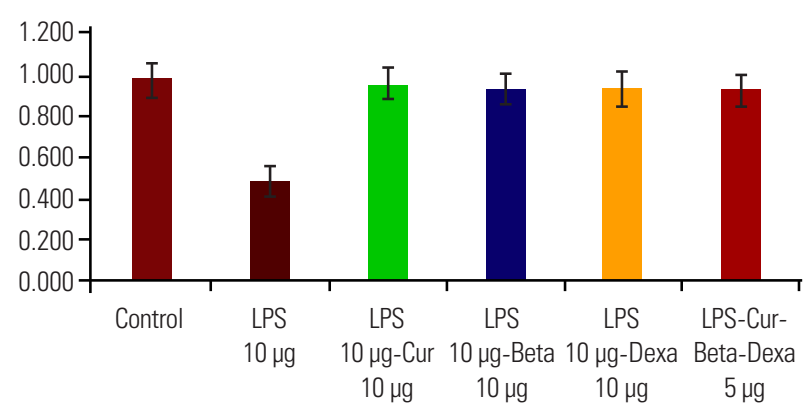

Fig. 3. MTT assay for assessing the cytotoxicity of individual and combined macrolactone and other steroid treatments on a normal cell line (human embryonic kidney-293). LPS, lipopolysaccharide; Cur, curvularin; Beta, betamethasone; Dexa, dexamethasone. effective at controlling MMP-2 and MMP-3 expression in NPCs compared to other protectants. NPCs treated with Beta, Dexa, and combinations showed variable expression of MMP-3.

ADAMTS are known as aggrecanases responsible for the degradation of ECM and loss of NPCs. ADAMTS-5 gene expression was significant in LPS-treated NPCs compared to Beta- and Dexa-treated NPCs; however, NPCs treated with Cur and combinations showed no expression of ADAMTS-5. The observed results indicate that Cur had been effective for controlling the actions of ADAMTS on aggrecanases in the ECM of NPC.

CYT-C is known as a death marker. CYT-C gene expression was observed for LPS treatment, at comparatively lower levels in Beta and Dexa treatment, and significantly lower in the combination treatment (Cur, Beta, and Dexa) groups. However, the expression of CYT-C was not observed in the control and Cur-treated groups. These results indicate the cytoprotective properties of Cur, Beta, and Dexa against LPS-induced cell death. Combined (Cur, Beta, and Dexa) treatment functioned synergistically with other treatments.

An anti-inflammatory response was initiated in LPS treatment of NPCs. As a result, we were able to observe the expression of anti-inflammatory molecules being activated or expressed. IL-2 and IL-10 are anti-inflammatory molecules, the expression of which was significant in LPS-treated NPCs, signifying their role in reducing LPSinduced effects [8-11]. The expression of IL-10 was not observed in the negative control nor in any of the other treated groups (Cur, Beta, and Dexa and their combination). The observations made in this experiment clearly indicate the efficiency of Cur, Beta, and Dexa at controlling LPS-induced inflammation, individually and in combination. Our results are supported by reported studies [8-11].

LPS induction initiates inflammation in NPCs. As a result, we observed the expression of repair genes being activated or overexpressed. IGF1R is an anti-injury or repair gene, the expression of which was significant in the LPStreated NPCs, signifying its role in reducing LPS-induced effects. The expression of IGF1R was not observed in the negative control, and also in NPCs treated with Cur, Beta, and Dexa individually, and in combination. The observations made in this experiment clearly indicate the efficiency of Cur, Beta, and Dexa at controlling LPS-induced inflammation, individually and in combination. 


\section{Expression of collagen genes in the experimental groups}

Collagen is part of the ECM. Collagen gene expression was lower in the LPS-treated group than in the other experimental groups, which indicated a loss of collagen. It was observed that Cur and combination treatments effected higher expression of collagen compared to steroid treatment (Fig. 4).

\section{Real-time polymerase chain reaction studies}

At a concentration of $10 \mu \mathrm{g} / \mathrm{mL}$, Cur and combination treatments downregulated the LPS-induced expression of proteolytic enzymes and pro-inflammatory markers in NPCs. As such, real-time quantitative-PCR results suggest that the upregulated expression of MMPs (MMP-2 and MMP-3) and ADAMTS (ADAMTS-5), induced by LPS, was downregulated by Cur and combination treatments. Similarly, LPS evidently increased the expression of proinflammatory markers (IL-1 $\beta$, IL-6); this increase was appreciably inhibited by Cur and combination treatments. A partial reversal of LPS-induced expression of collagen 9 by Cur and combination treatments was observed. Notably,

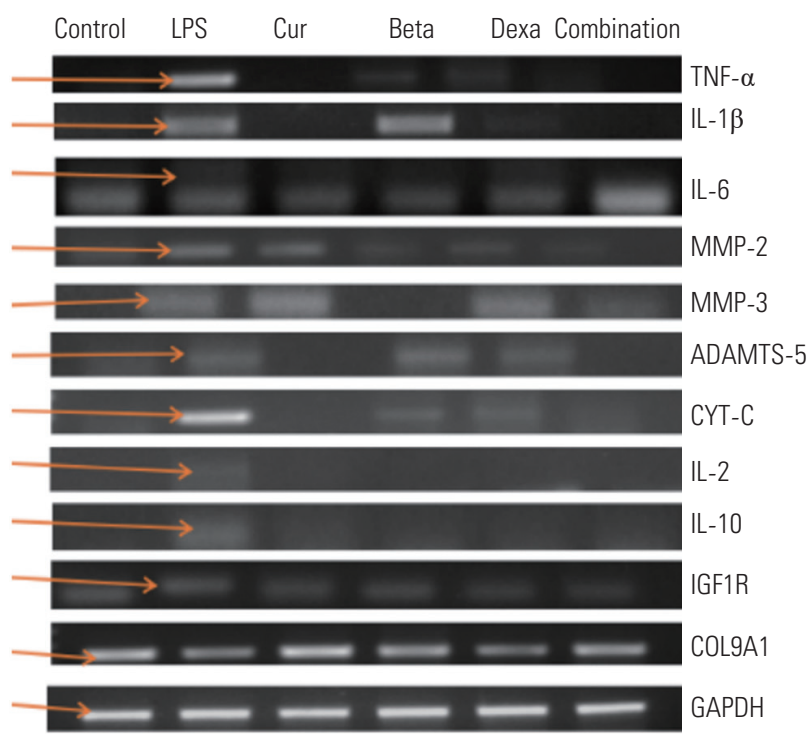

Fig. 4. Gene expression of pro-inflammatories (TNF- $\alpha$, IL-1 $\beta$, and IL-6), metalloproteases (MMP-2, MMP-3; ADAMTS-5), anti-inflammatories (IL-2 and IL10), cell death marker (CYT-C), stress-cum-repair marker (IGF1R), collagen 9A1, and endogenous control (GAPDH) in all experimental groups. TNF, tumor necrosis factor; IL, interleukin; MMPs, matrix metalloproteinases; CYT-C, cytochrome c; IGF1R, insulin-like growth factor 1 receptor; COL9A1, collagen 9 alpha 1; GAPDH, glyceraldehyde 3-phosphate dehydrogenas. the overexpression of IGF1R induced by LPS was also significantly suppressed by Cur and combination treatments.

The above results indicate the cytoprotective and antiinflammatory properties of Cur, Beta, and Dexa against LPS-induced cell death. Combination treatment worked synergistically with others (Cur, Beta, and Dexa).

\section{Discussion}

Perineural inflammation is a significant contributor to LBP and sciatica in patients with degenerative pathologies. Inflammation initiates the expression of proinflammatory cytokines, proteolytic enzymes (MMPs; i.e., MMP-2 and MMP-3), and ADAMTS (ADAMTS-5), contributing to disc pathologies $[2,3]$. Increased levels of inflammatory cytokines such as IL-1 $\beta$, IL-6, and TNF- $\alpha$, which are associated with IVDD in human and animal models, are believed to compromise the biomechanical properties of intervertebral discs, thereby promoting a degenerative state [2]. On one hand, IL-1 $\beta$ and TNF- $\alpha$, as two key mediators involved in degenerative changes in intervertebral discs, can bind to their membrane receptors and induce the downregulation of B-cell lymphoma 3 -encoded protein, thus promoting NPC apoptosis. On the other hand, IL-1 $\beta$ and TNF- $\alpha$ promote the expression of MMPs and inhibit the synthesis of collagen and proteoglycan as well as the proliferation of NPCs [1215].Corticosteroids and nonsteroidal anti-inflammatory drugs (NSAIDs) are often used to treat patients with LBP and sciatica [27]. Studies conducted by Benyamin et al. [28] in 2012 and Mailis and Taenzer [29] in 2012 reported that repeated administration of epidural injections affected bone mineral density, which leads to osteoporosis [30]. Additionally, NSAIDs inhibit the activity of cyclooxygenase- 2 and the synthesis of prostaglandin, as they play a role in the upregulation of inflammation [29,30]. Overexpression of TNF- $\alpha$ and IL- 6 by herniated discs was observed, which can induce the production of prostaglandin E2 [28]. The prolonged use of NSAIDS can induce gastrointestinal disturbances and renal and cardiac issues [30].

Cur is a novel bioactive fungal macrolactone (secondary metabolite) that can effect potent anti-inflammatory action; hence, it indicates importance for medicine and agriculture [22]. Schmidt et al. [21] in 2012 investigated the anti-inflammatory activity of (S)-Cur in mice and conducted microarray analyses of human C28/I2 chon- 


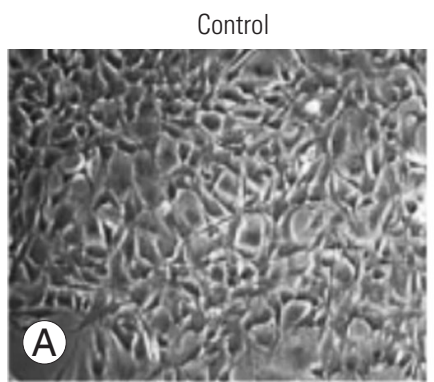

LPS-Beta $10 \mu \mathrm{g} / \mathrm{mL}$
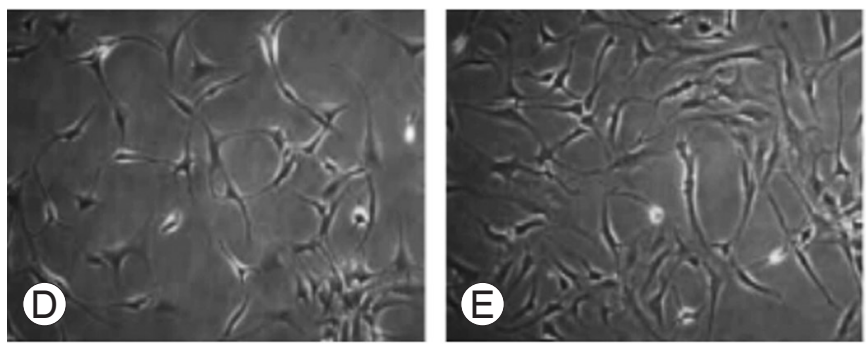

LPS $10 \mu \mathrm{g} / \mathrm{mL}$

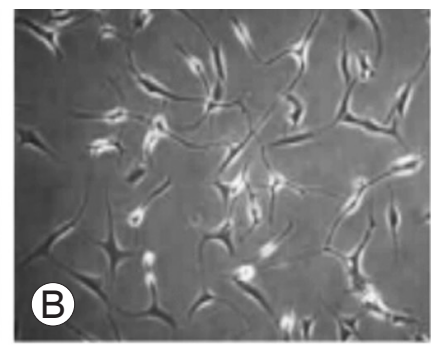

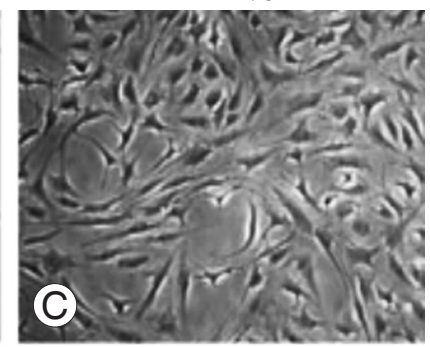

LPS-combination (Cur-Beta-Dexa) $10 \mu \mathrm{g} / \mathrm{mL}$

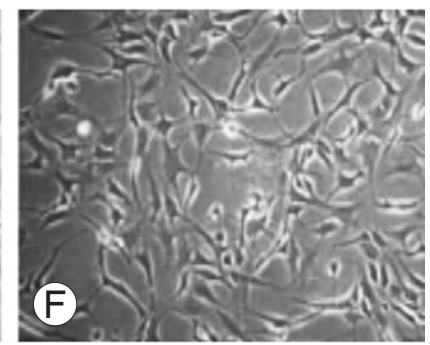

Fig. 5. (A-F) The effect of Cur, Beta, and Dexa, and their combination, on LPS-induced inflammation and loss of NPCs, compared to the untreated control group. We examined the growth enhancement and protective effects of Cur, Beta, and Dexa on NPCs. Cur, curvularin; NPCs, nucleus pulposus cells; LPS, lipopolysaccharide; Beta, betamethasone; Dexa, dexamethasone.

drocyte cells, by which they reduced the expression of pro-inflammatory genes. Nitric oxide (NO) plays a pivotal role in osteoarthritis and rheumatoid arthritis; (S)-Cur inhibited the production of $\mathrm{NO}$ by downregulating inducible NO synthase [22]. Rudolph et al. [16] in 2012 found that (S)-Cur and dehydrocurvularin can be used as anticancer drugs as they are able to hinder the cellular effects of transforming growth factor-beta, which is a key mediator in the cell cycle process.

The present study systematically evaluated the efficacy of Cur against LPS-induced inflammation in NPCs. Furthermore, we attempted to better understand the anti-inflammatory mechanism of Cur by studying the expression profile of inflammatory cytokines, metalloproteases, and apoptotic and structural genes in this in vitro research.

The fluorescence-activated cell sorting analysis conducted on isolated degenerated disk cells identified these cells as being NPCs, characterized using the CD24 marker (Fig. 2). The NPCs treated with LPS showed decreased cell numbers as well as cells that appeared to be blebbing or that were necrotic. However, cells that received Cur, Beta, and Dexa showed cytoprotective properties against LPSinduced cytotoxicity. NPCs that received Cur individually and in combination treatments exerted enhanced cytoprotective properties compared to individual Beta and Dexa treatments, which indicated that is a better cytoprotective agent (Figs. 5, 6). Similar findings were observed for

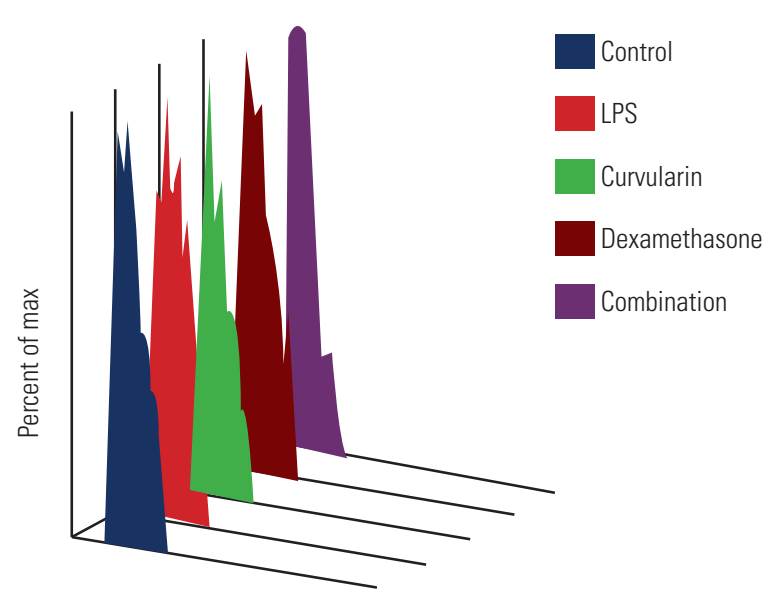

Fig. 6. The of individual and combinatorial anti-inflammators against LPS treated degenerated human NPC using cell proliferation assay. LPS, lipopolysaccharide.

the MTT assay, where treatment with LPS decreased cell viability but achieved the reversed result following treatment with protective agents (Cur, Beta, and Dexa), which indicated the cytoproliferative properties of these agents. The cytoprotective ability of Cur was found to be better compared to steroids (Fig. 3).

Gene expression studies (quantitative PCR and realtime quantitative-PCR) revealed the overexpression of pro-inflammatory cytokines (interleukins [IL- $1 \beta$ and IL-6] and TNF- $\alpha$ ), MMPs (MMP-2 and MMP-3), antiinflammatory (IL-2 and IL-10), and cell repair responses 

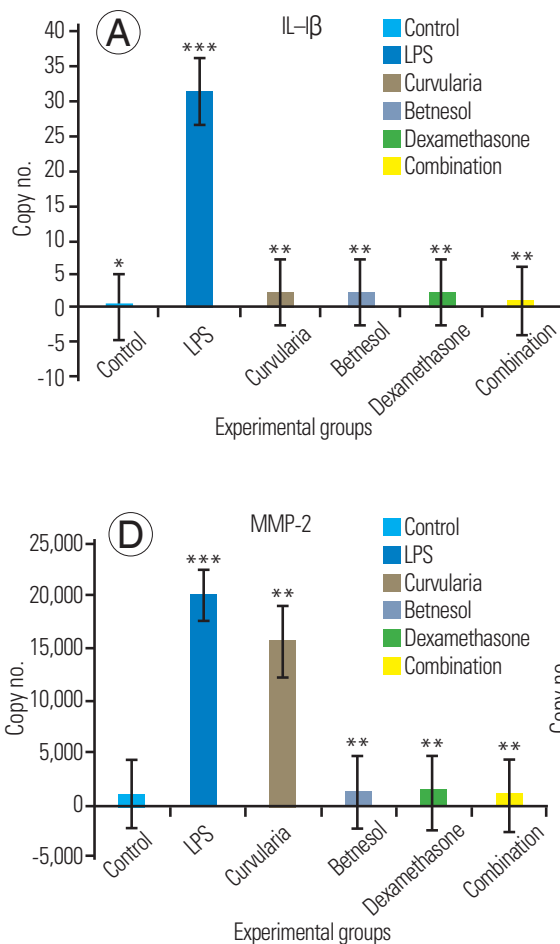

Experimental groups

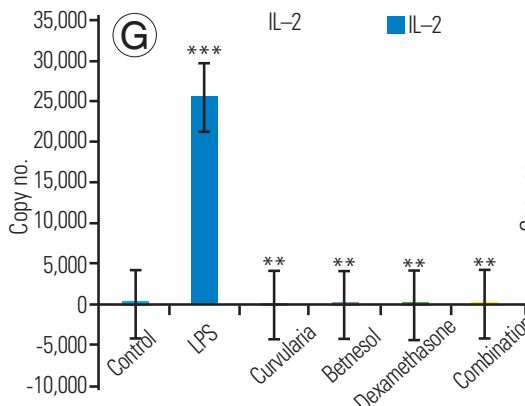

Experimental groups

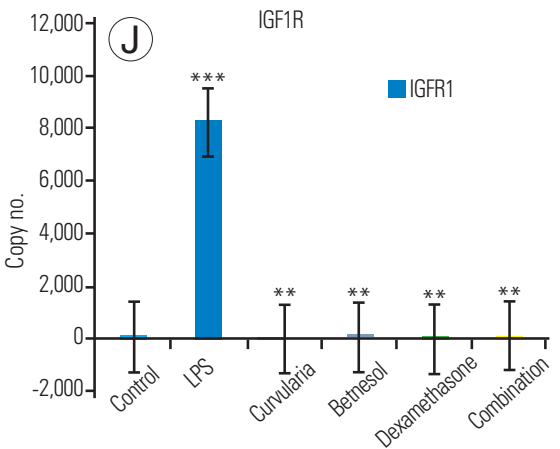

Experimental groups

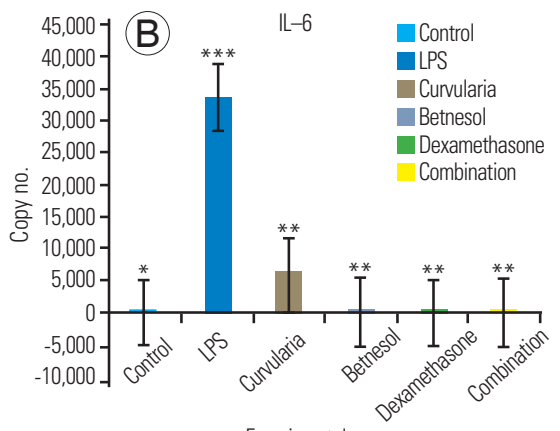

Experimental groups
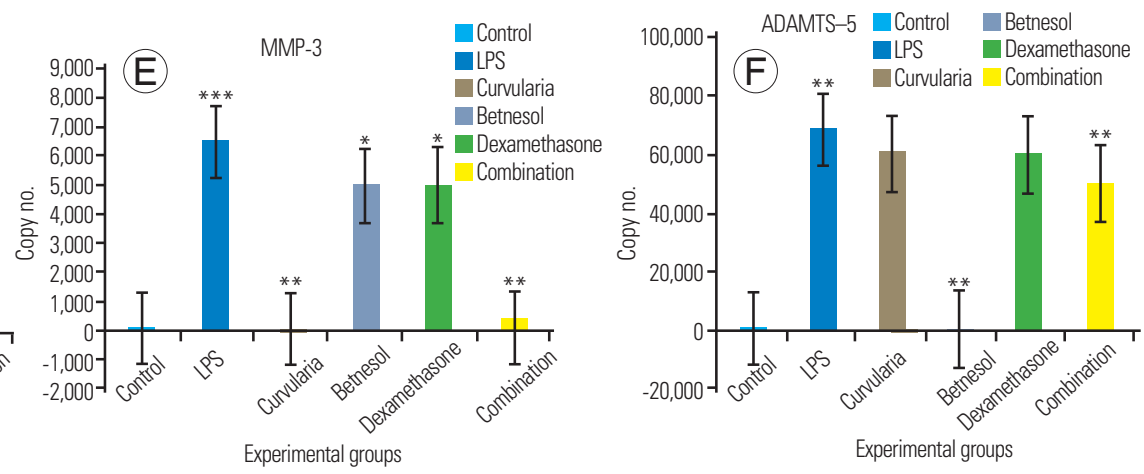

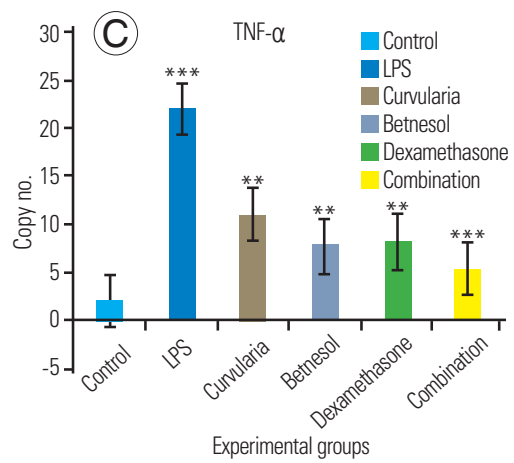

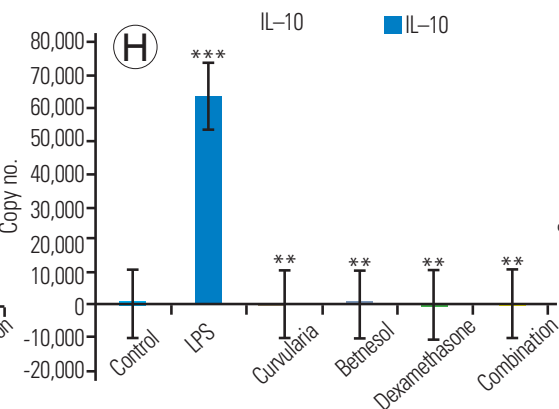

Experimental groups

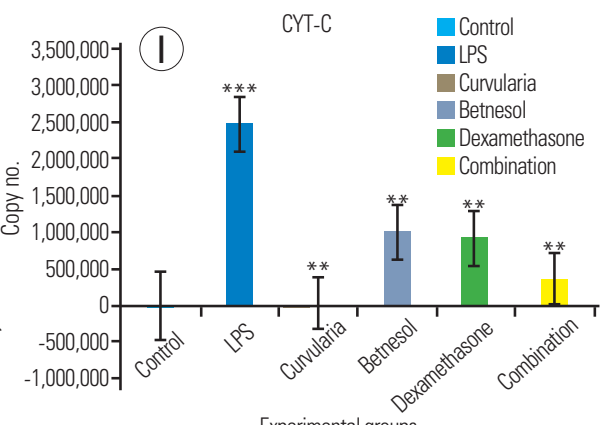

Experimental groups

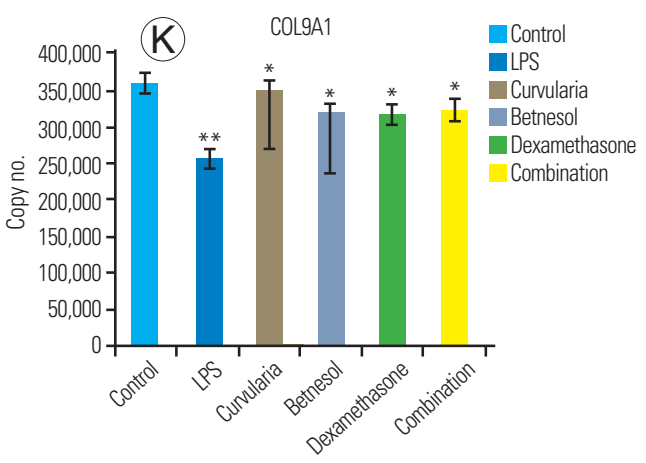

Experimental groups

Fig. 7. (A-K) Graphical representation of varied gene expressions using real-time polymerase chain reaction. LPS, lipopolysaccharide; TNF, tumor necrosis factor IL, interleukin; MMPs, matrix metalloproteinases; CYT-C, cytochrome c; IGF1R, insulin-like growth factor 1 receptor; COL9A1, collagen 9 alpha $1 .{ }^{*} p<0.05 .{ }^{* *} p<0.01$. ${ }^{* * *} p<0.001$.

(IGF1R); additionally, downregulation of the structural gene (collagen 9A1) of the ECM was observed in the degenerated human NPCs upon LPS treatment. Treat- ments with protective agents mediated the expression of pro-inflammatory cytokines (IL-1 $\beta$, IL- 6 , TNF- $\alpha$ ), metalloproteases (MMP-2 and MMP-3), IGF1R, and anti- 
Table 4. Real-time polymerase chain reaction studies showing the gene expression of various genes when treated with LPS and anti-inflammatories/cytoprotectants

\begin{tabular}{lcrrrrrrrrrr} 
& IL-1 $\beta$ & IL-6 & TNF- $\alpha$ & MMP-2 & MMP-3 & ADAMTS-5 & IL-2 & IL-10 & CYT-C & IGF1R & COL9A1 \\
Control & 0.0068 & 0.63 & 2.04 & $1,000.34$ & 0.68 & 0.27 & 0.04 & 0.14 & 0.05 & 1.63 & $35,997.90$ \\
LPS & $2,002.069$ & $33,456.5$ & $2,142.0562$ & $19,483.96$ & $4,544.79$ & $68,556.22$ & $254,975.65$ & $63,303.6$ & $2,476,721.12$ & $8,220.44$ & $256,789.00$ \\
Curvularin & 0.097 & $6,555.08$ & 11.05 & $15,545.16$ & 0.00 & $60,500.13$ & 0.00 & 0.00 & 0.00 & 0.00 & $348,942.77$ \\
Betamethasone & 0.085 & 2.63 & 7.87 & $1,300.01$ & $5,001.87$ & 0.01 & 0.01 & 0.32 & $990,000.19$ & 0.03 & $318,326.58$ \\
Dexamethasone & 0.080 & 0.92 & 8.21 & $1,350.13$ & $5,000.71$ & $60,000.34$ & 0.08 & 0.05 & $900,000.03$ & 0.08 & $316,970.68$ \\
Combination & 0.070 & 0.15 & 5.28 & $1,000.20$ & 0.25 & $50,000.11$ & 0.29 & 0.08 & $350,000.01$ & 0.24 & $32,894.56$ \\
\hline
\end{tabular}

LPS, lipopolysaccharide; IL, interleukin; TNF, tumor necrosis factor; MMPs, matrix metalloproteinases; CYT-C, cytochrome c; IGF1R, insulin-like growth factor 1 receptor; COL9A1, collagen 9 alpha 1 .

inflammatory genes (IL-2) and downregulated collagen 9A1 expression. The expression of IL-2 and IL-10 was only observed in the LPS-treated group, which indicated inflammatory stress. However, its absence clearly suggested the anti-inflammatory efficacy of protective agents (Fig. 6) [21]. Real-time PCR studies showed a higher copy number of pro-inflammatory cytokines (i.e., IL-1 $\beta$, IL6 , TNF- $\alpha$ ) and metalloproteases (MMP-2, MMP-3, and MMP-9) in the LPS-treated group (Fig. 7, Table 4). Gene expression studies have shown that Cur $(10 \mu \mathrm{g} / \mathrm{mL})$ exerts good anti-inflammatory and cytoproliferative properties on degenerating human NPCs when treated with $10 \mu \mathrm{g} /$ mL LPS in vitro. Treatments with Cur, Beta, and Dexa, individually and in combination, showed declined expression of inflammatory cytokines and metalloproteases in LPS-treated NPCs. The anti-inflammatory response of Cur was significantly better compared to steroid treatment.

\section{Conclusions}

In summary, the present study demonstrated that Cur exhibited anti-inflammatory, cytoprotective, and regenerative effects against LPS-stimulated NPCs by inhibiting inflammatory and metalloprotease mediators such as TNF- $\alpha$, IL-1 $\beta$, IL-6, MMP-2, MMP-3, and ADAMTS-5. Furthermore, Cur also showed signs of cell repair or regeneration, as it enhanced the expression of collagen 9A1 and IGF1R. Furthermore, we hypothesize that Cur may inhibit LPS-induced inflammatory and metalloprotease mediator production by obstructing the nuclear factor$\kappa \mathrm{B} /$ mitogen-activated protein kinase signaling pathway. Our study, for the first time, suggests the potential clinical application of Cur (i.e., an alternative to steroids and NSAIDs in pain management).
Future directions are as follows: (1) in vivo study followed by a large animal study; (2) potential for the development of a Cur-based formulation for epidural usage in the management of LBP and sciatica.

\section{Conflict of Interest}

No potential conflict of interest relevant to this article was reported.

\section{Acknowledgments}

We wish to thank our colleagues and Dr. A. Adarsh Reddy and Dr. H. Kushal for their continued support. I wish to thank Dr. Murahari Penkulinti for his support in editing the manuscript.

\section{References}

1. Banala RR, Vemuri SK, Dar GH, et al. Efficiency of dual siRNA-mediated gene therapy for intervertebral disc degeneration (IVDD). Spine J 2019;19:896-904.

2. Sampara P, Banala RR, Vemuri SK, Av GR, Gpv S. Understanding the molecular biology of intervertebral disc degeneration and potential gene therapy strategies for regeneration: a review. Gene Ther 2018;25:67-82.

3. Le Maitre CL, Pockert A, Buttle DJ, Freemont AJ, Hoyland JA. Matrix synthesis and degradation in human intervertebral disc degeneration. Biochem Soc Trans 2007;35:652-5.

4. Molinos M, Almeida CR, Caldeira J, Cunha C, Goncalves RM, Barbosa MA. Inflammation in intervertebral disc degeneration and regeneration. J R Soc Interface 2015;12:20141191. 
5. Devi YS, DeVine M, DeKuiper J, Ferguson S, Fazleabas AT. Inhibition of IL-6 signaling pathway by curcumin in uterine decidual cells. PLoS One 2015;10:e0125627.

6. Wuertz K, Quero L, Sekiguchi M, et al. The red wine polyphenol resveratrol shows promising potential for the treatment of nucleus pulposus-mediated pain in vitro and in vivo. Spine (Phila $\mathrm{Pa} 1976$ ) 2011;36:E1373-84.

7. Chanet A, Milenkovic D, Deval C, et al. Naringin, the major grapefruit flavonoid, specifically affects atherosclerosis development in diet-induced hypercholesterolemia in mice. J Nutr Biochem 2012;23:469-77.

8. Kawaii S, Tomono Y, Katase E, Ogawa K, Yano M. Antiproliferative effects of the readily extractable fractions prepared from various citrus juices on several cancer cell lines. J Agric Food Chem 1999;47:2509-12.

9. Li K, Li Y, Ma Z, Zhao J. Crocin exerts anti-inflammatory and anti-catabolic effects on rat intervertebral discs by suppressing the activation of JNK. Int J Mol Med 2015;36:1291-9.

10. Li Y, Li K, Hu Y, Xu B, Zhao J. Piperine mediates LPS induced inflammatory and catabolic effects in rat intervertebral disc. Int J Clin Exp Pathol 2015;8:6203-13.

11. Wang XH, Zhu L, Hong X, et al. Resveratrol attenuated TNF-a-induced MMP-3 expression in human nucleus pulposus cells by activating autophagy via AMPK/SIRT1 signaling pathway. Exp Biol Med (Maywood) 2016;241:848-53.

12. Le Maitre CL, Freemont AJ, Hoyland JA. The role of interleukin-1 in the pathogenesis of human intervertebral disc degeneration. Arthritis Res Ther 2005;7:R732-45.

13. Yu ZG, Xu N, Wang WB, Pan SH, Li KS, Liu JK. Interleukin-1 inhibits Sox9 and collagen type II expression via nuclear factor-kappaB in the cultured human intervertebral disc cells. Chin Med J (Engl) 2009;122:2483-8.

14. Wang J, Markova D, Anderson DG, Zheng Z, Shapiro IM, Risbud MV. TNF- $\alpha$ and IL- $1 \beta$ promote a disintegrin-like and metalloprotease with thrombospondin type I motif-5-mediated aggrecan degradation through syndecan-4 in intervertebral disc. J Biol Chem 2011;286:3973849.
15. Zhao CQ, Zhang YH, Jiang SD, Li H, Jiang LS, Dai LY. ADAMTS-5 and intervertebral disc degeneration: the results of tissue immunohistochemistry and in vitro cell culture. J Orthop Res 2011;29:718-25.

16. Rudolph K, Serwe A, Erkel G. Inhibition of TGF- $\beta$ signaling by the fungal lactones (S)-curvularin, dehydrocurvularin, oxacyclododecindione and galiellalactone. Cytokine 2013;61:28596.

17. Shen B, Melrose J, Ghosh P, Taylor F. Induction of matrix metalloproteinase- 2 and -3 activity in ovine nucleus pulposus cells grown in threedimensional agarose gel culture by interleukin1beta: a potential pathway of disc degeneration. Eur Spine J 2003;12:66-75.

18. Shen L, Wu Y, Han L, Zhang H. Overexpression of growth and differentiation factor-5 inhibits inflammatory factors released by intervertebral disc cells. Exp Ther Med 2018;15:3603-8.

19. Mancino R, Scro M, Frawley R, Grande DA, Bloom O, Chahine NO. The effect of lipopolysaccharide on cell viability and cytokine expression of bovine intervertebral disc cells. Proceedings of the 2011 Annual Meeting of the Orthopaedic Research Society: poster no. 719; 2011 Jan 13-16; Long Beach, USA. Rosemont (IL): Orthopaedic Research Society; 2011.

20. Kong JG, Park JB, Lee D, Park EY. Effect of high glucose on stress-induced senescence of nucleus pulposus cells of adult rats. Asian Spine J 2015;9:155-61.

21. Schmidt N, Art J, Forsch I, et al. The antiinflammatory fungal compound (S)-curvularin reduces proinflammatory gene expression in an in vivo model of rheumatoid arthritis. J Pharmacol Exp Ther 2012;343:106-14.

22. Ha TM, Ko W, Lee SJ, et al. Anti-inflammatory effects of curvularin-type metabolites from a marine-derived fungal strain penicillium sp. SF5859 in lipopolysaccharide-induced RAW264.7 macrophages. Mar Drugs 2017;15.

23. Kluba T, Niemeyer T, Gaissmaier C, Grunder T. Human anulus fibrosis and nucleus pulposus cells of the intervertebral disc: effect of degeneration and culture system on cell phenotype. Spine (Phila Pa 1976) 2005;30:2743-8. 
24. Devraj VM, Vemuri SK, Banala RR, Gunda SK, Av GR, Gpv S. Evaluation of anti-inflammatory and regenerative efficiency of naringin and naringenin in degenerated human nucleus pulposus cells: biological and molecular modeling studies. Asian Spine J 2019;13:875-89.

25. Vemuri SK, Banala RR, Subbaiah GP, Srivastava SK, Reddy AV, Malarvili T. Anti-cancer potential of a mix of natural extracts of turmeric, ginger and garlic: a cell-based study. Egypt J Basic Appl Sci 2017;4:332-44.

26. Vemuri SK, Banala RR, Mukherjee S, et al. Novel biosynthesized gold nanoparticles as anti-cancer agents against breast cancer: synthesis, biological evaluation, molecular modelling studies. Mater Sci Eng C Mater Biol Appl 2019;99:41729.
27. Briot K, Roux C. Glucocorticoid-induced osteoporosis. RMD Open 2015;1:e000014.

28. Benyamin RM, Manchikanti L, Parr AT, et al. The effectiveness of lumbar interlaminar epidural injections in managing chronic low back and lower extremity pain. Pain Physician 2012;15:E363-404.

29. Mailis A, Taenzer P. Evidence-based guideline for neuropathic pain interventional treatments: spinal cord stimulation, intravenous infusions, epidural injections and nerve blocks. Pain Res Manag 2012;17:150-8.

30. Quero L, Klawitter M, Nerlich AG, Leonardi M, Boos N, Wuertz K. Bupivacaine: the deadly friend of intervertebral disc cells? Spine J 2011;11:46-53. 